\title{
Pro-metastatic signaling of the trans fatty acid elaidic acid is associated with lipid rafts
}

\author{
SHINGO KISHI $^{1}$, RINA FUJIWARA-TANI ${ }^{1}$, YI LUO $^{2}$, ISAO KAWAHARA ${ }^{1}$, KEI GOTO ${ }^{1}$, KIYOMU FUJII ${ }^{1}$, \\ HITOSHI OHMORI ${ }^{1}$, CHIE NAKASHIMA ${ }^{1}$, TAKAMITSU SASAKI ${ }^{1}$ and HIROKI KUNIYASU ${ }^{1}$ \\ ${ }^{1}$ Department of Molecular Pathology, Nara Medical University, Kashihara, Nara 634-8521, Japan; \\ ${ }^{2}$ Jiangsu Province Key Laboratory of Neuroregeneration, Nantong University, Nantong, Jiangsu 226001, P.R. China
}

Received September 11, 2017; Accepted January 11, 2018

DOI: $10.3892 / \mathrm{ol} .2018 .7817$

\begin{abstract}
Trans fatty acids (TFAs) are risk factors for cardiovascular disorders, and the cancer-promoting effects of TFAs have been previously reported. The present study examined the effects and signaling of elaidic acid (EA), a TFA, in colorectal cancer (CRC) cells. Oral intake of EA was found to increase metastasis of HT29 human CRC cells. Results indicated that, in the plasma membrane, EA was integrated into cholesterol rafts, which contain epidermal growth factor receptors (EGFR). EA increased nanog and c-myc, and decreased PGC-1A through lipid raft-associated EGFR signaling in HT29 cells. Depletion of cholesterol by methyl- $\beta$-cyclodextrin treatment abrogated the EA-induced stemness and oxidative phosphorylation. Simvastatin treatment also abrogated EA-enhanced tumor growth. These results indicate that EA enhances the stemness by activating EGFR in lipid rafts.
\end{abstract}

\section{Introduction}

Colorectal cancer (CRC) is the third leading cause of cancer-related deaths in Japan (1), and its incidence has been reported to be increasing because of the popularity of western lifestyle $(2,3)$. We have previously reported the pro-tumoral effects of elaidic acid (EA), a major trans fatty acid (TFA) $(4,5)$. EA is a long-chain fatty acid (LCFA) containing 18 carbon chains in a trans configuration, and is a structural isomer of oleic acid (OA). EA promotes carcinogenesis in

Correspondence to: Professor Hiroki Kuniyasu, Department of Molecular Pathology, Nara Medical University, 840 Shijo-cho, Kashihara, Nara 634-8521, Japan

E-mail: cooninh@zb4.so-net.ne.jp

Abbreviations: CRC, colorectal cancer; TFA, trans fatty acid; LCFA, long-chain fatty acid; EA, elaidic acid; OA, oleic acid; $\mathrm{MbCD}$, methyl- $\beta$-cyclodextrin; EGF, epidermal growth factor; GPR, G-protein coupled receptor; EGF, epidermal growth factor; EGFR, EGF receptor; ERK, extracellular signal-regulated kinase; EMT, epithelial-mesenchymal transition; SIM, simvastatine

Key words: epidermal growth factor receptor, lipid raft, cholesterol
Min mice (6) and induces the proliferation of Ehrlich ascites sarcoma cells (7). EA enhances the metastasis of CRC in mouse models (4) by increasing cancer cell stemness and epithelial-mesenchymal transition (EMT) (5). EA also induces chemoresistance in CRC cells by upregulating its stemness (8).

LCFAs bind to specific membrane-bound receptors, namely, G-protein coupled receptor 40 (GPR40) and GPR120 (9). EA also binds to GPR40 and 120, inducing the transactivation of EGFR via c-src (5). Lipid rafts represent a special localization of epidermal growth factor receptors (EGFRs), altering its signaling pathways (10) and transactivation (11). Rao et al have reported that EA can affect Fas/FasL-induced apoptosis via lipid rafts (12). Lipid rafts may play an important role in EGFR signaling in EA-treated cells.

In the present study, we examined the effect of EA on pro-metastatic EGFR signals that may be associated with cholesterol rafts.

\section{Materials and methods}

Cell culture and reagents. HT29, a human colon cancer cell line, was purchased from Dainihon Pharmacy Co. (Tokyo, Japan). The cells were routinely maintained in Dulbecco's modified Eagle's medium (DMEM) supplemented with $10 \%$ fetal bovine serum (both Sigma, St. Louis, MO, USA) in $5 \% \mathrm{CO}_{2}$ at $37^{\circ} \mathrm{C}$. Cell morphology was evaluated daily by microscopic examination. Each cell line was routinely tested for Mycoplasma contamination by genomic PCR. The viability of each cell line was tested by trypan blue exclusion assay.

EA (CAS no. 112-79-8), OA (CAS no. 112-80-1; both WAKO Pure Chemicals, Osaka, Japan), and methyl- $\beta$-cyclodextrin (MbCD; Sigma) were purchased from the mentioned suppliers.

Cell membrane cholesterol was depleted by incubating the cells in a sterile-filtered MbCD medium (RPMI-1640 medium supplemented with $10 \mathrm{mM} \mathrm{MbCD}$ and cholesterol-free serum; Sigma).

Assessment of cell growth. The cells $\left(1 \times 10^{4}\right.$ per well) were seeded in a 12-well dish. Cell growth was assessed using a 3-(4,5-dimethylthiazol-2-yl)-2,5-diphenyltetrazolium bromide (MTT) assay or by counting the number of cells with an Autocytometer (Sysmex, Kobe, Japan) after 48 h, as described previously (13). 
Animal models. BALB/cSlc-nu/nu mice (males, 5 weeks old) were purchased from Japan SLC (Shizuoka, Japan). The mice were maintained according to the institutional guidelines approved by the Committee for Animal Experimentation of Nara Medical University (Kashihara, Japan), in accordance with the current regulations and standards established by the Ministry of Health, Labor, and Welfare. Each experimental group comprised 5 animals.

For the subcutaneous tumor model, HT29 cancer cells (1x10 ${ }^{7}$ cells) suspended in Hank's balanced salt solution (Sigma) were inoculated into the scapular subcutaneous tissue of mice. For analyzing the metastasis to the liver, lung, and peritoneum, cancer cells $\left(1 \times 10^{6}\right.$ cells) were inoculated into the spleen, the tail vein, or peritoneal cavity, respectively. EA or OA were administrated through a gavage. The growth of subcutaneous tumors and the metastatic status of the cells was assessed after 4 weeks. For assessing growth of subcutaneous tumors, the diameters of the tumors were measured every week. For peritoneal metastasis, the weight of mesenterium with tumors was measured. For metastases to the liver and lung, the number of tumors at the organ surface was counted. Simvastatin $(50 \mathrm{mg} / \mathrm{kg} / \mathrm{day}$; WAKO) was administrated through a gavage for 10 days after the inoculation of cancer cells.

Western blot analysis. Whole-cell lysates were prepared as described previously (14). Cell fractions were extracted by processing the cells with the Cell Fractionation kit (Abcam, Cambridge, MA, USA), according to the manufacturer's instructions.

Proteins $(20 \mu \mathrm{g})$ were separated from the cell lysates by sodium dodecyl sulfate-polyacrylamide gel electrophoresis on $12.5 \%$ gels. They were then electrotransferred onto nitrocellulose membranes for immunoblotting analysis. The membranes were incubated with primary antibodies, followed by incubation with peroxidase-conjugated $\operatorname{IgG}$ antibodies (Medical \& Biological Laboratories Co., Ltd, Nagoya, Japan). A tubulin antibody (Oncogene Research Products, Cambridge, MA, USA) was used to measure the amount of proteins loaded in each lane. Immune complexes were visualized using the CSA system (DAKO, Carpinteria, CA, USA). Antibodies against EGFR (Transduction Laboratories, Lexington, KY, USA), phosphorylated EGFR, phosphorylated ERK1/2 $\left(\mathrm{pThr}^{204}\right.$ ), nanog (clone 1E6C4), c-Myc (clone 9E10; Santa Cruz Biotechnology, Santa Cruz, CA, USA), phosphorylated p38 (pThr ${ }^{180} / \mathrm{pTy}^{182}$; Bioss Inc., Woburn, MA, USA), peroxisome proliferator-activated receptor $\gamma$ coactivator-1 $\alpha$ (PGC-1A; Proteintech Group, Inc., Rosemount, IL, USA) were used as primary antibodies.

Extraction of raft fractions. Lipid rafts were isolated from $5 \times 10^{7}$ cells. Briefly, cell pellets disrupted in $1 \mathrm{ml}$ buffer A [50 mM Tris- $\mathrm{HCl}$ ( $\mathrm{pH} 8.0$ ), $10 \mathrm{mM} \mathrm{MgCl}, 0.15 \mathrm{M} \mathrm{NaCl}$, $1 \%$ Triton $\mathrm{X}-100,5 \%$ glycerol, $50 \mathrm{mM}$ PMSF, $1 \mathrm{x}$ protease inhibitor cocktail (Sigma), and $0.03 \% \beta$-mercaptoethanol] were centrifuged for $5 \mathrm{~min}$ at $500 \mathrm{x} \mathrm{g}$ and $4^{\circ} \mathrm{C}$. The obtained supernatant was added to $1 \mathrm{ml}$ buffer A [50 mM Tris- $\mathrm{HCl}$ (pH 8.0), $10 \mathrm{mM} \mathrm{MgCl} 2,0.15 \mathrm{M} \mathrm{NaCl}$, and $80 \%$ sucrose] to make up the final concentration of sucrose to $40 \%$. A discontinuous sucrose gradient was obtained by stratifying $7.5 \mathrm{ml}$ buffer A with $38 \%$ sucrose and $2 \mathrm{ml}$ buffer A with $15 \%$ sucrose. The gradient was ultracentrifuged for $18 \mathrm{~h}$ at $100,000 \mathrm{x}$ g and $4^{\circ} \mathrm{C}$.

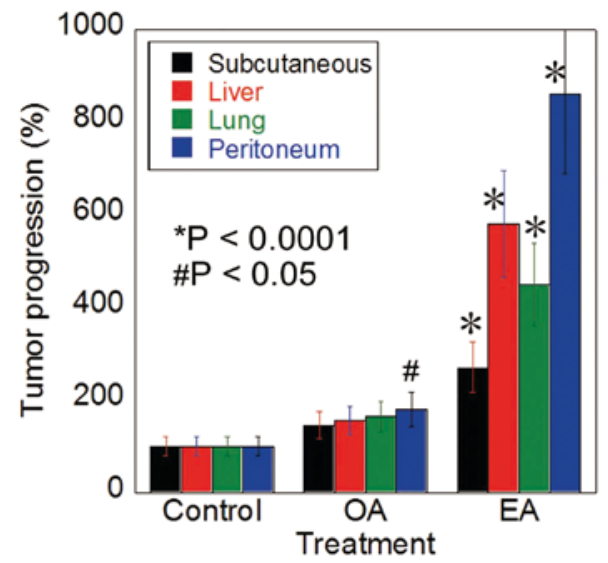

Figure 1. Oral intake of EA enhanced the metastasis of CT26 cells. Metastasis of HT29 human CRC cells in nude mice treated with EA, OA $(10 \mathrm{mg} / \mathrm{mouse}$ in $30 \%$ ethanol), or vehicle (30\% ethanol) by gavage administration. Tumors were assessed 4 weeks after cancer cell inoculation by counting the number of tumors at the liver surface. Error bars are the mean \pm SD. ${ }^{*} \mathrm{P}<0.0001$ and ${ }^{\#} \mathrm{P}<0.05$ vs. control. CRC, colorectal cancer; EA, elaidic acid; OA, oleic acid.

Twelve fractions ( $1 \mathrm{ml}$ each) were collected from the top to the bottom of the gradient (F1-F12). Immunoblotting analysis was performed to identify the fractions containing lipid rafts. Cholesterol levels were measured using the Amplex Red cholesterol assay kit (Thermo Fisher Scientific, Inc., Waltham, MA, USA).

Statistical analysis. Statistical analyses of experimental data were performed using the Mann-Whitney $U$ test or the Kruskal-Wallis test with Dunn's multiple comparison (nonparametric ANOVA). Differences with a two-sided P-value $<0.05$ were considered to indicate a statistically significant difference.

\section{Results}

EA enhances metastasis. The HT29 cells were inoculated into the subcutaneous tissue, spleen (liver metastasis), tail vein (lung metastasis), and peritoneum of nude mice treated with EA, OA, or control vehicle by gavage administration (Fig. 1). The EA-treated mice showed higher growth of subcutaneous tumors and metastases to liver, lungs, and peritoneum than control mice. In contrast, only peritoneal metastasis was increased in the OA-treated mice, compared to the control mice.

EA activates EGFR in lipid rafts. We examined the integration of EA into lipid rafts (Fig. 2A). We observed that EA was preferentially integrated into cholesterol rafts, significantly increasing their cholesterol content (Fig. 2B). In contrast, $\mathrm{MbCD}$ preferentially decreased the cholesterol content of EA-induced rafts (Fig. 2A). EA integration into cholesterol rafts upregulated the accumulation and induced the phosphorylation of EGFR (Fig. 2B). In contrast, MbCD suppressed EA-induced phosphorylation of EGFR and ERK1/2 (Fig. 2C, D). Furthermore, the levels of phosphorylated p38 were higher than the levels of phosphorylated ERK1/2 in cholesterol-raft depleted cells (Fig. 2D). 

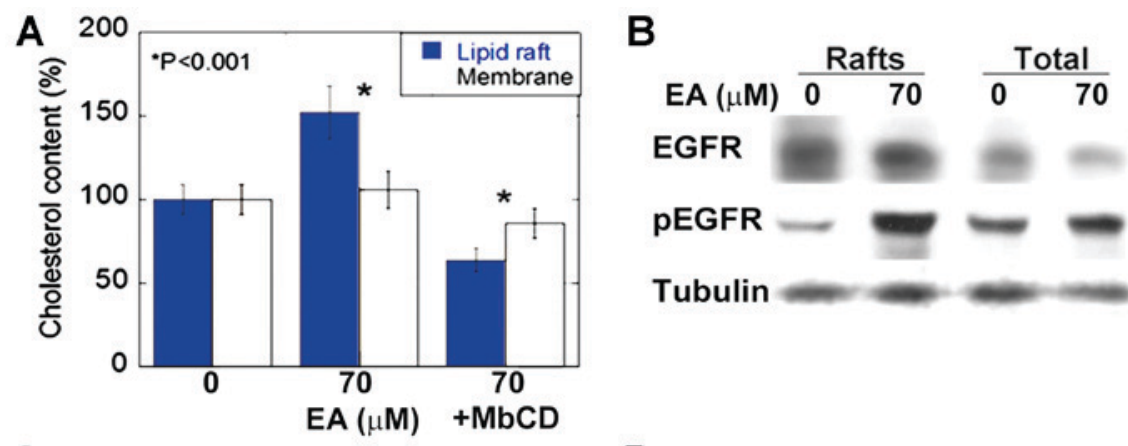

Tubulin
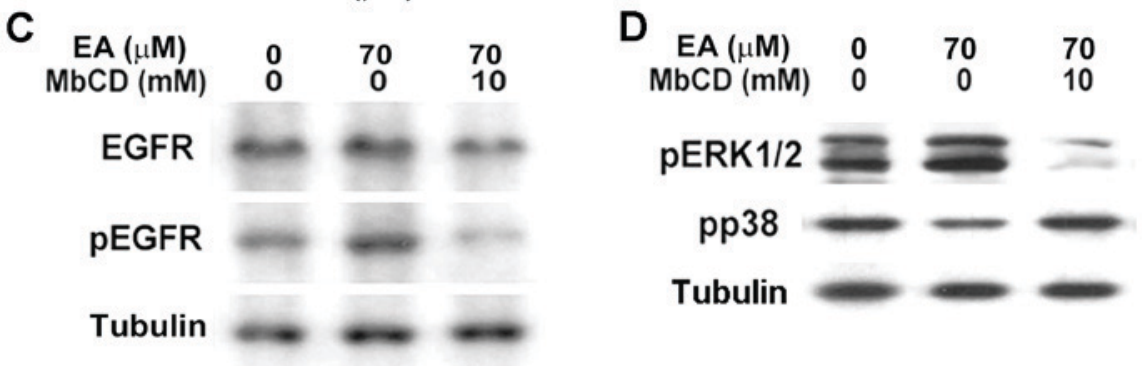

Figure 2.Effect of EA on EGFR activation in lipid rafts.(A) Effect of EA on cholesterol content in total membrane or detergent-resistant raft fractions." $\mathrm{P}<0.001$ vs. 0 group. (B) EGFR and phosphorylated EGFR levels in lipid rafts or membrane fractions in CT26 cells. (C) Effect of MbCD on EA-induced EGFR phosphorylation. (D) Effect of MbCD on EA-induced ERK1/2 and p38 phosphorylation in CT26 cells. Tubulin was used as an internal control. Each bar or point represents mean \pm SD of three independent experiments. EA, elaidic acid; EGFR, epidermal growth factor receptor; MbCD, methyl- $\beta$-cyclodextrin; $\mathrm{pERK1/2,}$ phosphorylated ERK1/2; pp38, phosphorylated p38.
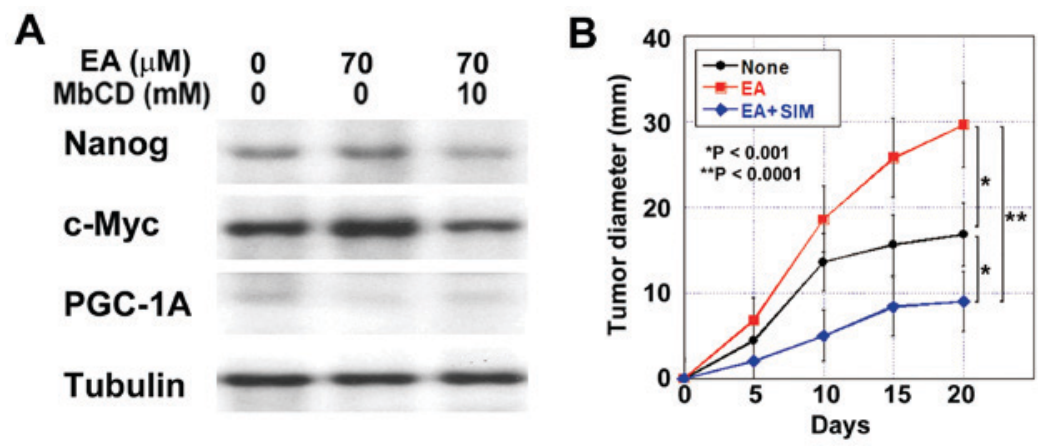

Figure 3. Effect of EA, with or without SIM, on stemness and energy metabolism. (A) Protein expression levels of nanog, c-Myc, and PGC-1A in HT29 cells treated with EA or EA and MbCD. Tubulin was used as an internal control. (B) Effect of simvastatin on tumor growth of HT29 subcutaneous tumors in nude mice treated with EA. SIM $(20 \mathrm{mg} / \mathrm{kg} /$ day $)$ was administrated with a gavage for 10 days after the inoculation. Error bars are the mean \pm SD. EA, elaidic acid; SIM, simvastatin; PGC-1A, proliferator-activated receptor $\gamma$ coactivator-1 $\alpha$.

Effect of EA on cancer stem cell-proteins. Next, we examined the role of lipid rafts on the stemness of cancer cells, which is known to be enhanced by EA (5). EA treatment increased nanog production in HT29 cells; however, it was abrogated by cholesterol depletion after MbCD treatment (Fig. 3A). c-Myc protein levels were also increased by EA treatment and abrogated by cholesterol depletion. In contrast, PGC-1A levels were decreased by EA and abrogated by cholesterol depletion.

Simvastatine, which has been reported to deplete lipid rafts in a cholangiocarcinoma mouse model (15), was administrated to the EA-treated mice. In the subcutaneous HT29 cell tumor model, lipid raft depletion inhibited the EA-induced tumor growth (Fig. 3B). The tumor growth rate in the simvastatine and EA-treated mice was lower than that in the EA-treated mice and even than that in the control mice.

\section{Discussion}

Results of the present study indicated that EA could strongly enhance cancer metastasis by increasing their stemness via EGFR activation in lipid rafts.

In our data, HT29 cells showed enhanced metastasis after EA gavage administration. EA activated EGFRs, as reported in our previous report (5); however, it was important that EA-induced EGFR signals disappeared after the absorbance of cholesterol, which caused lipid raft depletion. Raft depletion also abrogated the EA-induced expression of nanog and c-myc. These findings suggested that EA-associated signal pathways may prefer lipid rafts.

EA can be incorporated into plasma membranes composed of stearic or palmitic acid, to be subsequently converted into triglycerides (16), cholesterol esters, phospholipids, or other LCFAs such as OA and LA (17). Our results were consistent 
with the findings of these studies; we observed that EA could easily integrate into the plasma membrane, especially cholesterol rafts, of CRC cells.

We found that lipid rafts played an important role in EA signaling. Lipid rafts are microdomains within the lipid bilayer of cellular membranes. Levels of non-constitutive proteins in lipid rafts fluctuate dramatically in different cancers, affecting various signaling pathways (18). We have previously reported that EA binds to GPR40 and GPR120, which signals SRC-mediated crosstalk to EGFRs (5). Another previous study had shown that the crosstalk between GPRs and EGFRs in the raft could activate phospholipases C, D, and A2, which could activate protein kinase C, ERK, and Akt (19). Lipid rafts are known to be thicker than the surrounding membrane and the propensity of EGFR to be located in rafts is characterized by a thick lipid bilayer (35.0 ̊̊) around the transmembrane segment of the receptor (20).

The raft-associated EA signal upregulated nanog and c-Myc, while it downregulated PGC-1A. c-Myc is associated with cell proliferation and aerobic glycolysis, and PGC-1A is associated with stemness and oxidative phosphorylation $(21,22)$. The upregulation of nanog in the present data and our previous data (5) indicated that EA increased the stemness of cancer cells; however, the cancer cells may have been of a proliferative nature with glycolytic energy metabolism, which is different from dormant stem cells that possess oxidative phosphorylation metabolism. The induction of proliferative cancer stem cells may be the most striking characteristic of EA to enhance cancer metastasis.

We observed that cholesterol depletion inhibited EA-enhanced tumor growth below the level of untreated tumors in the subcutaneous tumor mouse model. Importantly, lipid raft depletion may inhibit EGFR signals in the presence or absence of EA. Statins have been reported to inhibit the growth of JAK2-mutated cells by inhibiting lipid rafts (23) and inducing apoptosis by inhibiting Rac1 (15). Thus, lipid rafts act as a platform for collecting multiple stimulants, receptors, and signals to activate cancer progression (24). The mechanism of integration of EA to cholesterol raft should be elucidated in further examinations; however, our data suggests that lipid rafts may be promising targets for anticancer treatment.

\section{Acknowledgements}

The authors thank Ms. Tomomi Masutani for assistance with the preparation of this manuscript. The present study was supported by MEXT KAKENHI (grant nos. 16H05164, 17K15648, 17K19923 and 16K19087).

\section{References}

1. Wakao F, Nishimoto $\mathrm{H}$, Kataonoda $\mathrm{K}$, Tsukuma $\mathrm{H}$ and Mikami H (eds.): Cancer Statistics in Japan, 2013. National Cancer Res Inst, Tokyo, 2013.

2. Kimura Y, Kono S, Toyomura K, Nagano J, Mizoue T, Moore MA, Mibu R, Tanaka M, Kakeji Y, Maehara Y, et al: Meat, fish and fat intake in relation to subsite-specific risk of colorectal cancer: The Fukuoka colorectal cancer study. Cancer Sci 98: 590-597, 2007.

3. Mizoue T, Tanaka K, Tsuji I, Wakai K, Nagata C, Otani T, Inoue $\mathrm{M}$ and Tsugane S; Research Group for the Development and Evaluation of Cancer Prevention Strategies in Japan: Alcohol drinking and colorectal cancer risk: An evaluation based on a systematic review of epidemiologic evidence among the Japanese population. Jpn J Clin Oncol 36: 582-597, 2006.
4. Ohmori H, Fujii K, Kadochi Y, Mori S, Nishiguchi Y, Fujiwara R, Kishi S, Sasaki T and Kuniyasu H: Elaidic acid, a trans-fatty acid, enhances the metastasis of colorectal cancer cells. Pathobiology 84: 144-151, 2017.

5. Fujii K, Luo Y, Fujiwara-Tani R, Kishi S, He S, Yang S, Sasaki T, Ohmori $\mathrm{H}$ and Kuniyasu H: Pro-metastatic intracellular signaling of the elaidic trans fatty acid. Int J Oncol 50: 85-92, 2017.

6. Molin M, Berstad P, Benth JS, Alexander J, Paulsen JE and Almendingen $\mathrm{K}$ : Effect of different degrees of hydrogenated fish oil on intestinal carcinogenesis in Min/+ mice. Anticancer Res 33: 477-483, 2013.

7. Awad AB: Trans fatty acids in tumor development and the host survival. J Natl Cancer Inst 67: 189-192, 1981.

8. Tanabe E, Kitayoshi M, Fujii K, Ohmori H, Luo Y, Kadochi Y, Mori S, Fujiwara R, Nishiguchi Y, Sasaki T and Kuniyasu H: Fatty acids inhibit anticancer effects of 5-fluorouracil in mouse cancer cell lines. Oncol Lett 14: 681-686, 2017.

9. Hara T, Kashihara D, Ichimura A, Kimura I, Tsujimoto G and Hirasawa A: Role of free fatty acid receptors in the regulation of energy metabolism. Biochim Biophys Acta 1841: 1292-1300, 2014.

10. Zhang Z, Wang L, Du J, Li Y, Yang H, Li C, Li H and Hu H: Lipid raft localization of epidermal growth factor receptor alters matrix metalloproteinase-1 expression in $\mathrm{SiHa}$ cells via the MAPK/ERK signaling pathway. Oncol Lett 12: 4991-4998, 2016.

11. Overmiller AM, McGuinn KP, Roberts BJ, Cooper F, Brennan-Crispi DM, Deguchi T, Peltonen S, Wahl JK III and Mahoney MG: c-Src/Cav1-dependent activation of the EGFR by Dsg2. Oncotarget 7: 37536-37555, 2016.

12. Rao H, Ma LX, Xu TT, Li J, Deng ZY, Fan YW and Li HY: Lipid rafts and Fas/FasL pathway may involve in elaidic acid-induced apoptosis of human umbilical vein endothelial cells. J Agric Food Chem 62: 798-807, 2014.

13. Kuniyasu H, Yano S, Sasaki T, Sasahira T, Sone S and Ohmori H: Colon cancer cell-derived high mobility group 1/amphoterin induces growth inhibition and apoptosis in macrophages. Am J Pathol 166: 751-760, 2005.

14. Kuniyasu H, Oue N, Wakikawa A, Shigeishi H, Matsutani N, Kuraoka K, Ito R, Yokozaki $\mathrm{H}$ and Yasui W: Expression of receptors for advanced glycation end-products (RAGE) is closely associated with the invasive and metastatic activity of gastric cancer. J Pathol 196: 163-170, 2002.

15. Miller T, Yang F, Wise CE, Meng F, Priester S, Munshi MK, Guerrier M, Dostal DE and Glaser SS: Simvastatin stimulates apoptosis in cholangiocarcinoma by inhibition of Rac1 activity. Dig Liver Dis 43: 395-403, 2011.

16. Harvey KA, Walker CL, Xu Z, Whitley P and Siddiqui RA: Trans fatty acids: induction of a pro-inflammatory phenotype in endothelial cells. Lipids 47: 647-657, 2012.

17. Vidgren HM, Louheranta AM, Agren JJ, Schwab US and Uusitupa MI: Divergent incorporation of dietary trans fatty acids in different serum lipid fractions. Lipids 33: 955-962, 1998.

18. Staubach S and Hanisch FG: Lipid rafts: Signaling and sorting platforms of cells and their roles in cancer. Expert Rev Proteomics 8: 263-277, 2011.

19. Rozengurt E: Mitogenic signaling pathways induced by G protein-coupled receptors. J Cell Physiol 213: 589-602, 2007.

20. Postic G, Ghouzam Y, Etchebest C and Gelly JC: TMPL: A database of experimental and theoretical transmembrane protein models positioned in the lipid bilayer. Database (Oxford) 2017, 2017.

21. Sancho P, Burgos-Ramos E, Tavera A, Bou Kheir T, Jagust P, Schoenhals M, Barneda D, Sellers K, Campos-Olivas R, Graña O, et al: MYC/PGC-1 $\alpha$ balance determines the metabolic phenotype and plasticity of pancreatic cancer stem cells. Cell Metab 22: 590-605, 2015.

22. Scognamiglio R, Cabezas-Wallscheid N, Thier MC, Altamura S, Reyes A, Prendergast ÁM, Baumgärtner D, Carnevalli LS, Atzberger A, Haas S, et al: Myc depletion induces a pluripotent dormant state mimicking diapause. Cell 164: 668-680, 2016.

23. Griner LN, McGraw KL, Johnson JO, List AF and Reuther GW: JAK2-V617F-mediated signalling is dependent on lipid rafts and statins inhibit JAK2-V617F-dependent cell growth. Br J Haematol 160: 177-187, 2013.

24. Irwin ME, Mueller KL, Bohin N, Ge Y and Boerner JL: Lipid raft localization of EGFR alters the response of cancer cells to the EGFR tyrosine kinase inhibitor gefitinib. J Cell Physiol 226: 2316-2328, 2011. 